\title{
An epigenomic approach to therapy for tamoxifen-resistant breast cancer
}

Qin Feng ${ }^{1}$, Zheng Zhang ${ }^{1}$, Martin J Shea ${ }^{2}$, Chad J Creighton ${ }^{3}$, Cristian Coarfa ${ }^{3}$, Susan G Hilsenbeck ${ }^{2}$, Rainer Lanz ${ }^{1}$, Bin $\mathrm{He}^{1,4}$, Lei Wang ${ }^{1}$, Xiaoyong $\mathrm{Fu}^{1,2,3}$, Agostina Nardone, ${ }^{1,3}$, Yongcheng Song ${ }^{5}$, James Bradner ${ }^{6}$, Nicholas Mitsiades $^{3,4}$, Constantine S Mitsiades ${ }^{6}$, C Kent Osborne ${ }^{1,2,3}$, Rachel Schiff ${ }^{1,2,3}$, Bert W O’Malley ${ }^{1,3}$

${ }^{I}$ Department of Molecular and Cellular Biology, Baylor College of Medicine, Houston, TX 77030, USA; ${ }^{2}$ Lester \& Sue Smith Breast Center, Baylor College of Medicine, Houston, TX 77030, USA; ${ }^{3}$ Dan L. Duncan Cancer Center, Baylor College of Medicine, Houston, TX 77030, USA; ${ }^{4}$ Department of Medicine-Hematology \& Oncology, Baylor College of Medicine, Houston, TX 77030, USA; ${ }^{5}$ Department of Pharmacology, Baylor College of Medicine, Houston, TX 77030, USA; ${ }^{6}$ Department of Medical Oncology, Dana-Farber Cancer Institute, Department of Medicine, Harvard Medical School, Boston, MA 02215, USA

Tamoxifen has been a frontline treatment for estrogen receptor alpha (ER $\alpha)$-positive breast tumors in premenopausal women. However, resistance to tamoxifen occurs in many patients. ER still plays a critical role in the growth of breast cancer cells with acquired tamoxifen resistance, suggesting that ER $\alpha$ remains a valid target for treatment of tamoxifen-resistant (Tam-R) breast cancer. In an effort to identify novel regulators of ERo signaling, through a small-scale siRNA screen against histone methyl modifiers, we found WHSC1, a histone H3K36 methyltransferase, as a positive regulator of ERo signaling in breast cancer cells. We demonstrated that WHSC1 is recruited to the ER $\alpha$ gene by the BET protein BRD3/4, and facilitates $E R \alpha$ gene expression. The small-molecule BET protein inhibitor JQ1 potently suppressed the classic ERo signaling pathway and the growth of Tam-R breast cancer cells in culture. Using a Tam-R breast cancer xenograft mouse model, we demonstrated in vivo anti-breast cancer activity by JQ1 and a strong long-lasting effect of combination therapy with JQ1 and the ER degrader fulvestrant. Taken together, we provide evidence that the epigenomic proteins BRD3/4 and WHSC1 are essential regulators of estrogen receptor signaling and are novel therapeutic targets for treatment of Tam- $R$ breast cancer.

Keywords: epigenomic; tamoxifen; breast cancer

Cell Research (2014) 24:809-819. doi:10.1038/cr.2014.71; published online 30 May 2014

\section{Introduction}

Estrogen signaling is crucial for the development of normal mammary gland and breast cancer. Estrogen binds to and activates estrogen receptors (ERs), resulting in expression of genes involved in cell proliferation and survival. By blocking estrogen binding to ER alpha $(\mathrm{ER} \alpha)$, the selective ER modulator, tamoxifen remains a frontline treatment for ER $\alpha$-positive breast cancer [1]. However, eventually many tumors develop tamoxifen resistance. Interestingly, $\mathrm{ER} \alpha$ is still important for the

Correspondence: Bert W O’Malley

E-mail: berto@bcm.edu

Received 20 April 2014; revised 24 April 2014; accepted 28 April 2014; published online 30 May 2014 growth of breast cancer cells with acquired drug resistance. For instance, recent chromatin immunoprecipitation (ChIP)-seq analysis on primary breast tumors from patients clearly shows that ER $\alpha$ is still recruited to chromatin in drug-resistant breast cancer [2]. Moreover, in the absence of estrogen, epidermal growth factor could induce AP-1-dependent ER $\alpha$ genomic targets [3]. Therefore, there is an urgent need to develop novel treatments to further suppress ER signaling in Tam-R breast cancer. Recent data show that mTOR inhibition is effective in overcoming hormone resistance [4]. However, mTOR inhibition is associated with several side effects, limiting its use in patients who must take it for a long time. Combinations of HDAC inhibitor vorinostat and tamoxifen also showed some effect in reversing hormone resistance [5]. In this study, we are exploring a novel strategy to overcome endocrine resistance by shutting down expres- 
sion of the $E R$ gene itself.

\section{Results}

\section{WHSC1 regulates ERa gene expression}

Recent progress has shown that histone-modifying enzymes and coregulators play important roles in the regulation of gene expression [6-8]. In this study, we were interested in understanding how histone modifications, particularly histone methylation, regulate ER $\alpha$ signaling in breast cancer cells. To this end, we performed a smallscale siRNA screen to identify epigenomic enzymes involved in estrogen signaling. We knocked down histone methyltransferases (HMTs) or demethylases (HDMTs) individually in MCF7 cells by siRNA, and determined the expression levels of ER $\alpha$ and its target genes GREB1 and $p S 2$. We focused on the HMTs and HDMTs that target lysine residues, because the diverse roles of histone lysine methylation in gene expression have been well established. The role of 29 HMTs and 18 HDMTs in estrogen signaling was tested by knockdown using individual siRNAs. Supplementary information, Figure S1 shows the mRNA levels of ER $\alpha$ and its target gene GREB1. Knockdown of SMYD3 led to an increased level of GREB1 mRNA, while knockdown of three HMTs, ASH1L, SETD7 and WHSC1, and two HDMTs, KDM4A and KDM7C reduced GREB1 mRNA level by
$>60 \%$. Similar effects were observed for $p S 2$ gene expression (data not shown). ER $\alpha$ mRNA levels were also significantly reduced in these samples, indicating that these epigenomic enzymes are critical for ER $\alpha$ and its target gene expression. We confirmed the reduction of ER $\alpha$ protein levels by western blot analysis as shown in Figure 1A.

WHSC1 encodes a HMTs that methylates histone H3K36 [9, 10]. Methylation of H3K36 is a key histone mark for transcription elongation. WHSC1 was initially found to be deleted in Wolf-Hirschhorn syndrome (WHS), which is a malformation syndrome associated with a hemizygous deletion of the distal short arm of chromosome 4. Later studies revealed that WHSC1 is significantly overexpressed in many cancers including breast cancer $[11,12]$. Importantly, its expression is associated with tumor aggressiveness and bad prognosis in several breast cancer studies $[13,14]$. However, the underlying molecular mechanism of its role in breast cancer development remains unknown. Therefore, we focused on WHSC1 for further study.

Using three individual WHSC1 siRNAs, we confirmed the essential role of WHSC1 in expression of $\mathrm{ER} \alpha$ and its target genes (Figure 1B and 1C). Interestingly, WHSC1 mRNA levels were increased by two-fold in MCF7 cells treated with estradiol, indicating that WHSCl itself is an ER $\alpha$-regulated gene (Figure 1D), forming a positive
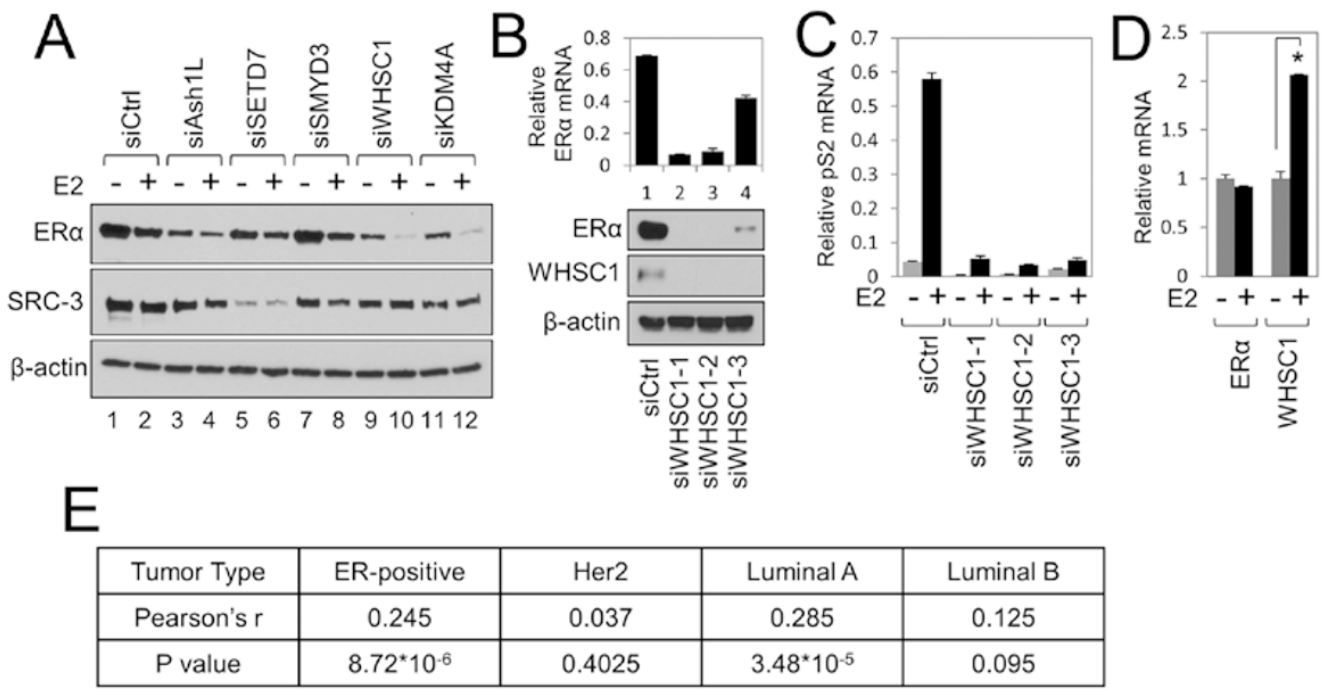

Figure 1 WHSC1 regulates ER $\alpha$ gene expression. (A) Confirmation of siRNA screening results by western blot analysis. Five genes were selected from the siRNA screening. The protein levels of ER $\alpha$ and SRC-3 were measured by western blot analysis. (B) Knockdown of WHSC1 by three different siRNAs all decreased mRNA and protein levels of ER $\alpha$ in MCF7 cells. (C) Knockdown of WHSC1 reduced the expression of ER $\alpha$ target gene $p S 2$ in MCF7 cells. $10 \mathrm{nM}$ of estradiol (E2) was added $24 \mathrm{~h}$ before cell harvest. (D) Enhanced expression of WHSC1 by treatment of $10 \mathrm{nM} \mathrm{E2}$ for $24 \mathrm{~h}$ in MCF7 cells. mRNA levels of ER $\alpha$ and WHSC 1 were measured by RT-qPCR. ${ }^{*} P<0.05$ by a two-tailed $t$-test. (E) Correlation of ER $\alpha$ mRA and WHSC1 mRNA levels in a subset of the TCGA breast cancer samples. 
feedback regulatory loop. In support of this, we found a positive correlation in mRNA levels between WHSC1 and $\mathrm{ER} \alpha$ in ER-positive patients in the TCGA database, particularly in luminal-A-type of breast tumors (Figure 1E).

WHSC1 and BRD3/4 coordinately regulate ERa expression and function

Because WHSC1 does not contain a DNA-binding domain, it cannot bind directly to the ER $\alpha$ gene promoter/ enhancer to regulate its transcription. To investigate how WHSC 1 activates ER $\alpha$ gene expression, we searched the Epicome database (http://epicome.org), a mass spectrometry-based proteomics database generated by the Nuclear Receptor Signaling Atlas (NURSA) $[15,16]$, to identify WHSC1-interacting proteins. BRD3 and BRD4, two bromodomain-containing proteins, are at the top of a list of potential WHSC1-interacting proteins (Supplementary information, Figure S2A). Their association with WHSC1 was confirmed by immunoprecipitation/western blot analysis using either exogenously expressed or endogenous proteins. As shown in Figure 2A-2C, BRD3 and BRD4 were co-immunoprecipitated with WHSC1 protein and vice versa, but another BET family protein, BRD2, did not interact significantly with WHSC1. Similar interaction was detected between endogenous BRDs and WHSC1 in MCF7 cells (Supplementary information, Figure S2), and this is in agreement with a recent report of an interaction between WHSC1 and BRD4 in MEF cells [17].

BRD3 and BRD4 belong to the BET (bromodomain and extraterminal domain) family of proteins. In humans, there are four BET proteins including BRD2, BRD3, BRD4, and BRDT [18]. BET family members contain two bromodomain and one extraterminal domain. The bromodomain specifically recognizes acetylated lysine residues on the histone tails. BRD3 and BRD4 are implicated in the transcription elongation process by association with the PAF1 complex and the pTEFb complex, respectively [19]. BRD4 also regulates the expression of G1 cell cycle genes [20]. We next determined the domain responsible for the interaction between WHSC1 and BRD3/4 by deletion mapping. As shown in Supplementary information, Figure S2D and S2E, BRD4 interacts with WHSC1 through its N-terminal 470 amino acids, which contains two bromodomains. Interestingly, treatment of cells with JQ1 failed to disrupt the interaction between WHSC1 and BRD4, suggesting that BRD4 can bind to WHSC1 and acetylated lysine simultaneously (Supplementary information, Figure S2F).

Given the key role of WHSC1 in ER $\alpha$ gene expression and the physical association between WHSC1 and
BRD3/4, we hypothesized that WHSC1 is recruited to the ER $\alpha$ gene promoter by BRD3/4 which binds directly to acetylated histone tails and subsequent methylation of $\mathrm{K} 36$ on histone $\mathrm{H} 3$ by WHSC1, then could facilitate the transcription elongation of the $E R \alpha$ gene. To test this hypothesis, we asked whether BRD3/4 are required for the expression of ER $\alpha$ and its target genes. As shown in Figure 2D, simultaneous knockdown of BRD3 and BRD4 dramatically reduced the mRNA levels of ER $\alpha$ and $p S 2$, indicating that BRD3 and BRD4 are crucial for the expression of ER $\alpha$.

To further test the above hypothesis, BRD3 and BRD4 genes were knocked down in MCF7 cells, and the recruitment of WHSC1 to the ER $\alpha$ gene and the level of histone H3 K36 methylation were determined by ChIP-qPCR assay. As shown in Figure 2E, knockdown of BRD3/4 dramatically reduced the recruitment of WHSC1 to the ER $\alpha$ gene and the levels of H3K36me2 and H3K36me3 were both significantly reduced, suggesting that BRD3/4 functions as a scaffold to recruit WHSC1, which promotes the transcription elongation of the $E R \alpha / E S R 1$ gene.

In our model shown in Figure 2F, BET proteins (BRD3/4) recognize acetylated lysine residues on histone tails in the ERo/ESR 1 gene promoter. After recruitment to the $E R \alpha$ gene by interacting with BRD3/4, WHSC1 methylates $\mathrm{H} 3 \mathrm{~K} 36$ and subsequently increases transcription elongation of ER $\alpha$. On the other hand, estrogen stimulates the expression of WHSC1, forming a positive feedback regulatory loop. Interestingly, we also found that WHSC1 and BRD4 are potent coactivators for ER in an ERE-luciferase gene reporter assay (Supplementary information, Figure S3A). Taken together, our results indicate that WHSC1 is a key regulator of ER signaling, as it is not only a positive epigenomic regulator of ER gene expression, but also a coactivator for ER itself.

Small-molecule inhibitors have been recently developed and published for the BET family of proteins. For instance, JQ1, an acetylated lysine analog, has been reported in recent studies to be a potent BET inhibitor and can be used to treat a number of cancers including multiple myeloma and acute myeloid leukemia [21-23]. Based on our results, we tested whether JQ1 can suppress ER $\alpha$ expression. As shown in Figure 2G, treatment of MCF7 cells with JQ1 significantly reduced the mRNA levels of ER $\alpha$ and its target genes $p S 2, G R E B 1$, and Cyclin D1, but not other breast cancer genes such as FoxA1, SRC-3, and Her2 (Supplementary information, Figure S3B). Another bromodomain inhibitor I-BET had a similar effect, although at a slightly higher concentration (Figure 2H). Similar experiments were performed on prostate cancer LNCaP cells. Interestingly, androgen receptor mRNA 
A
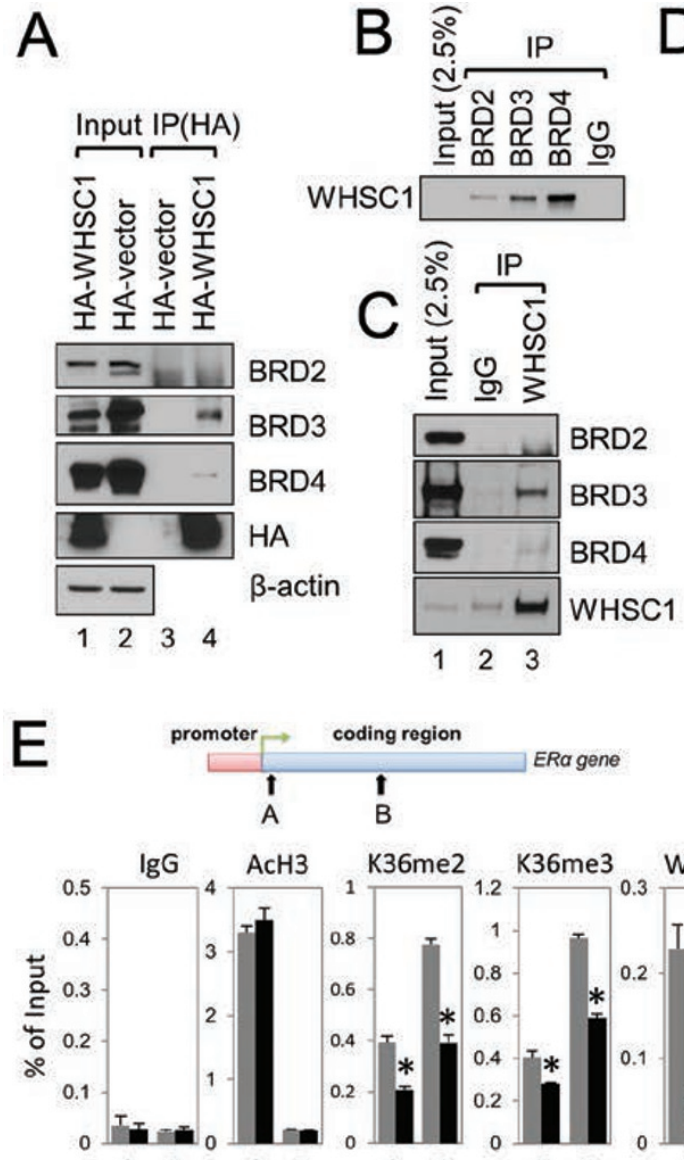

A B
B
D
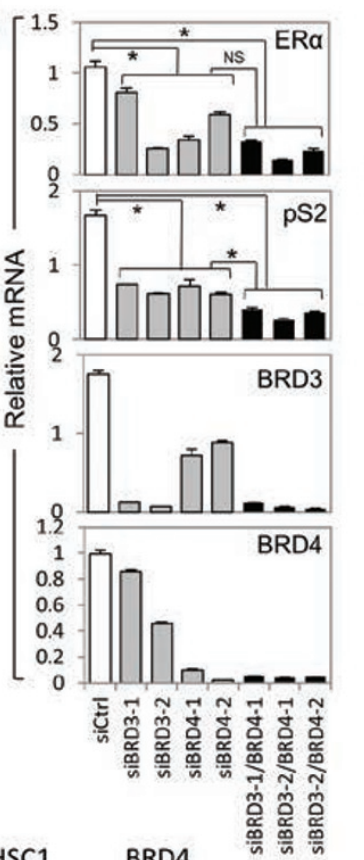
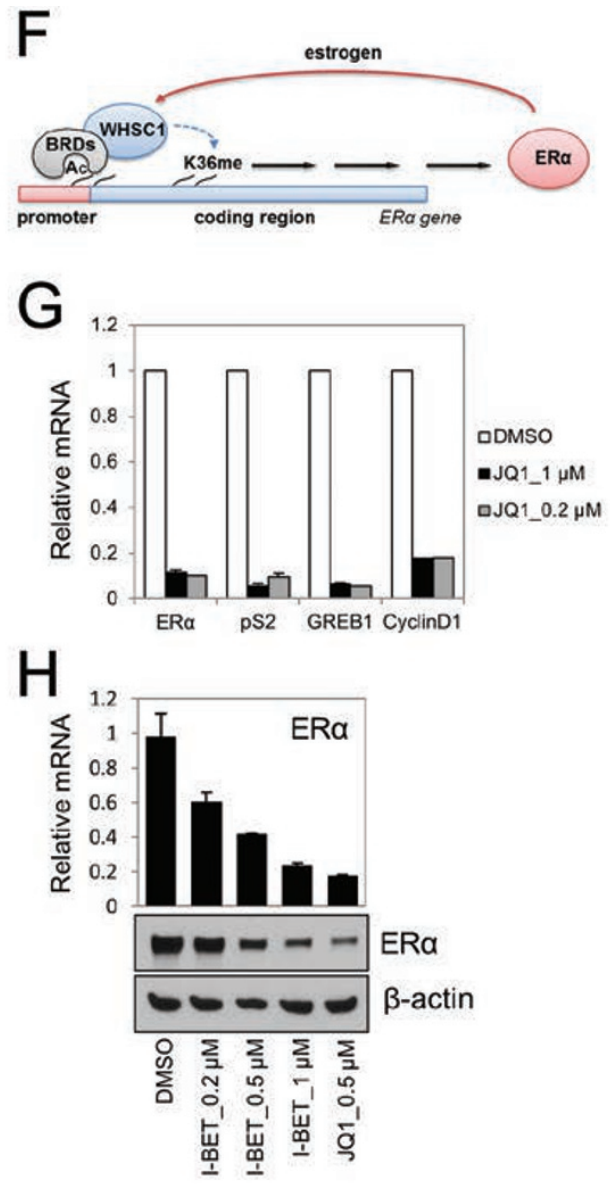

Figure 2 WHSC1 and BRD3/4 coordinately regulate ER $\alpha$ expression and function. (A) Co-immunoprecipitation (co-IP) of WHSC1 and BRD proteins from transiently transfected 293T cells. HA-tagged WHSC1 or empty vector was transiently transfected to $293 \mathrm{~T}$ cells for $48 \mathrm{~h}$. The whole-cell lysate was cleared and immunoprecipitated with anti-HA antibody. Input, 2.5\%. (B) Co-IP of endogenous WHSC1 and BRD proteins from HeLa nuclear extract. Input, $2.5 \%$. (C) Reciprocal co-IP of endogenous WHSC1 and BRD proteins from HeLa nuclear extract. Input, 2.5\%. (D) Knockdown of BRD3 and BRD4 reduced expression of ER $\alpha$ and its target gene $p S 2$ in MCF7 cells. Error bars indicate SEM. * $P<0.01$ by $t$-test. (E) Loss of BRD3/4 abolished the recruitment of WHSC1 to ER $\alpha$ gene. ChIP was performed in MCF7 cells treated with BRD3/4 siRNA or control siRNA for 2 days. Primer pair A locates next to promoter region and primer pair B locates in the coding region of ER $\alpha$. Each IP was duplicated and average values were shown. Error bars indicate SEM. ${ }^{*} P<0.05$ by $t$-test. (F) A hypothetical model of regulation of ER $\alpha$ gene expression by WHSC1 and BRD3/4. (G) Bromodomain inhibitor JQ1 efficiently reduced the expression of ER $\alpha$ and its target genes in MCF7 cells. Cells were treated with JQ1 for $24 \mathrm{~h}$ before harvest. $(\mathbf{H})$ Another Bromodomain inhibitor I-BET had similar function in suppressing ER $\alpha$ expression. MCF7 cells were treated with I-BET for $24 \mathrm{~h}$ before harvest.

levels were not altered upon JQ1 treatment, indicating that the suppression of ER $\alpha$ expression by JQ1 is selective (Supplementary information, Figure S3C).

\section{JQ1 inhibits growth of Tam-R breast cancer cells}

Next, we investigated whether the BRD3/4 inhibitor JQ1 inhibits growth of breast cancer cells. We treated parental MCF7 cells and a Tam-R derivative with different doses of JQ1 or vehicle control. As shown in Figure 3A, JQ1 potently inhibited the growth of Tam-R MCF7 cells, while it only moderately inhibited the growth of parental MCF7 cells at a concentration of $0.2 \mu \mathrm{M}$ (At a higher concentration of $0.5 \mu \mathrm{M}$, JQ1 also inhibited parental MCF7 cell growth.). We further tested three more ER-positive breast cancer cell lines including T47D, MCF7 RN, and ZR75-1 cells. We found that JQ1 inhibited the growth of all of these breast cancer cell lines, with higher efficacy toward the Tam-R cells. Interestingly, JQ1 also inhibited four estrogen-deprivation-resistant (mimics aromatase inhibitor resistance) lines (Supplementary information, Figure S4A). Simlar to MCF7 cells, JQ1 downregulated ER $\alpha$ mRNA levels in MCF7 
RN and ZR75-1 cells. The ER $\alpha$ dominant-negative splicing variant ER $\Delta 7$ was similarly downregulated [24] (Supplementary information, Figure S4B and S4C).

Our ChIP-qPCR analysis provided further evidence that disruption of BRD3/4/WHSC1/ER $\alpha$ axis by JQ1 suppressed ER $\alpha$ gene expression. As shown in Figure $3 \mathrm{~B}$, overall, the levels of histone modifications marking active promoters, such as acetylated histone $\mathrm{H} 3$ and $\mathrm{H} 3 \mathrm{~K} 4 \mathrm{me} 3$, were significantly lower on the ER $\alpha$ gene promoter in Tam-R MCF7 cells, likely due to lack of other key transcriptional factor(s). JQ1 treatment in Tam-R cells eliminated the recruitment of BRD3/4 and WHSC1 to the ER $\alpha$ gene promoter, and dramatically reduced the level of H3K4me3 and H3K36me3, two histone marks of transcription activation. In parental MCF7 cells, the recruitment of BRD3/4 and WHSC1 was still maintained at relatively high levels, although it was reduced by JQ1. BRD3 and BRD4 mRNA levels were not reduced (actually increased) by JQ1, while WHSC1 was reduced by JQ1 both at the mRNA and protein levels (Figure 3C and 3D).
We next wished to understand why Tam-R breast cancer cells are more sensitive to JQ1. Knockdown of WHSC1 alone reduced the ER $\alpha$ mRNA levels similarly in both parental and Tam-R breast cancer cells, and inhibited the growth of parental and Tam-R MCF7 cells similarly (Supplementary information, Figure S4D and S4E). This result suggests that WHSC1 is an important regulator of ER $\alpha$ gene expression and cell growth, but does not cause extra JQ1 sensitivity in Tam-R cells. However, we noticed that when cells were treated with JQ1 for up to 3 days, ER $\alpha$ mRNA was persistantly suppressed in Tam-R MCF7 cells (Figure 4A). In contrast, in parental MCF7 cells, ER $\alpha$ mRNA level was abolished initially, but recovered after prolonged treatment (Figure 4A). Moreover, JQ1 was reported to inhibit MYC signaling in previous studies $[21,25,26]$. Thus, we measured expression of MYC in JQ1-treated MCF7 cells. As shown in Figure 4A, MYC mRNA level responded to JQ1 treatment similarly to ER in parental and Tam-R MCF7 cells. These results demonstrate that ER $\alpha$ and MYC are JQ1 target genes in Tam-R MCF7 cells, and that sustained sup-
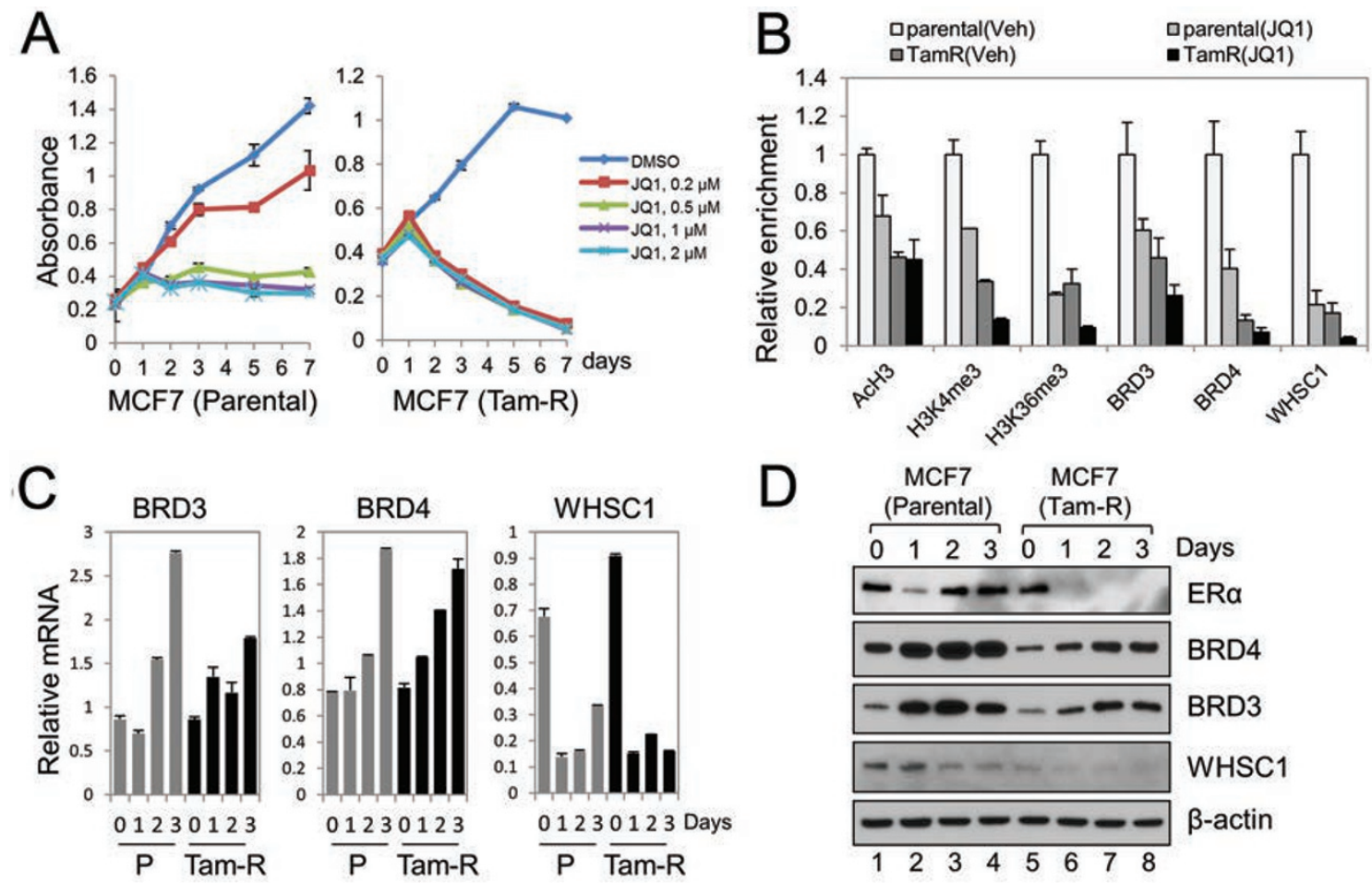

Figure 3 JQ1 inhibits growth of Tam-R cells. (A) JQ1 efficiently inhibited the growth of Tam-R MCF7 breast cancer cells as determined by MTS assay. Error bars indicate SEM. (B) ChIP assay to determine the level of histone modifications and recruitment of BRD3/4 and WHSC1 to ER $\alpha$ promoter. MCF7 parental and Tam-R cells were treated with DMSO (Veh) or $0.2 \mathrm{M}$ of JQ1 for $24 \mathrm{~h}$ before cell harvest. Primer Pair A (shown in Figure 2E) was used for qPCR analysis. Error bars were shown as SEM. (C) Expression of BRDs and WHSC1 in $0.2 \mu \mathrm{M}$ JQ1-treated MCF7 cells. (D) Western blot analysis of ER $\alpha$, BRDs, and WHSC1 proteins from $0.2 \mu \mathrm{M}$ JQ1-treated MCF7 cells. 

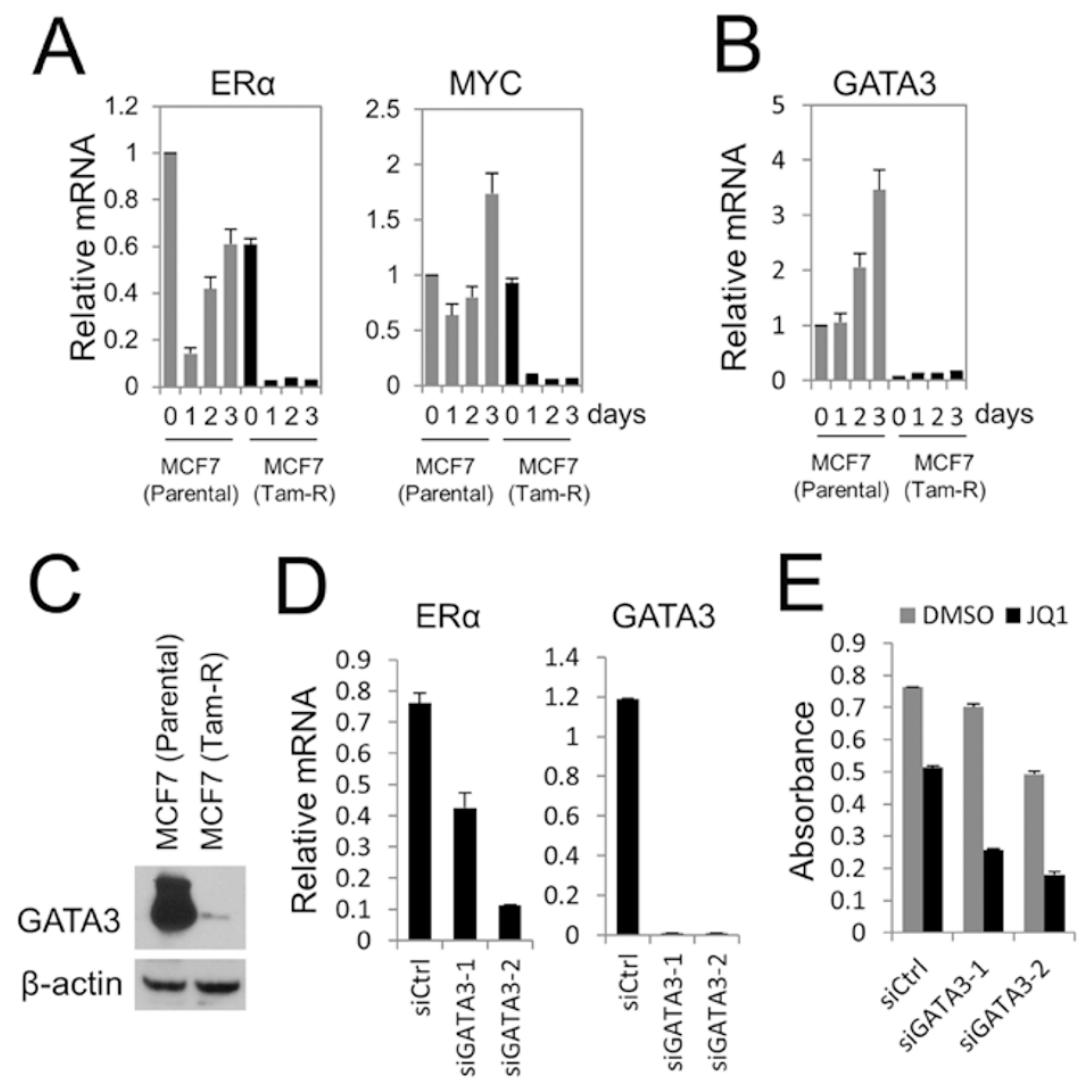

Figure 4 GATA3 is a potential factor to regulate JQ1 sensitivity in MCF7 cells. (A) JQ1 suppressed both ER $\alpha$ and MYC signaling pathways in Tam-R MCF7 cells. Parental and Tam-R MCF7 cells were treated with $0.2 \mu \mathrm{M}$ of JQ1 for different days, and mRNA levels of $E R \alpha$ and MYC were analyzed by RT-qPCR. (B) Expression of GATA3 in MCF7 parental and Tam-R cells after JQ1 treatment. (C) Comparison of GATA3 protein levels by western blot analysis in MCF7 parental and Tam-R cells. (D) Knockdown of GATA3 by siRNA reduces ER $\alpha$ gene expression. (E) Knockdown of GATA3 by siRNA enhances JQ1 inhibition function in MCF7 parental cells. All error bars were shown as SEM. pression of ER $\alpha$ and MYC by JQ1 probably contributes to its more potent anticancer activity on Tam-R breast cancer cells. To gain more mechanistic insight into this observation, we found that GATA3, a key regulator of ER gene expression [27], is highly expressed in parental MCF7 cells, but not in Tam-R cells (Figure 4B and 4C). In parental MCF7 cells, GATA3 expression is further increased by JQ1 treatment (Figure 4B). When we knocked down GATA3 using siRNA, the parental MCF7 cells became more sensitive to JQ1 treatment (Figure 4E). Thus, our results suggest that other key transcription factors, such as GATA3 in parental MCF7 cells, could have contributed to the JQ1 resistance with prolonged treatment. A decrease in such factors (Figure 4B) might contribute to epigenomic environmental changes on the ER $\alpha$ promoter, resulting in greater JQ1 sensitivity in Tam-R lines.

To determine the global signaling pathways that are altered by JQ1 in addition to ER and MYC, microarray analysis was performed on Tam-R MCF7 cells treated with vehicle or $0.2 \mu \mathrm{M}$ of JQ1. When applying a threshold of $\log 2<-0.2$ or $\log 2>0.2$, we identified 652 downregulated genes and 219 upregulated genes in JQ1-treated cells (Figure 5A). Supplementary information, Table S1A lists all the genes upregulated or downregulated in major biological pathways by KEGG pathway analysis.
Figure 5B shows the biological pathways negatively affected by JQ1. Among them, the cell cycle is an important pathway being affected since cell cycle-related gene expression was significantly altered by JQ1 treatment (Supplementary information, Figure S5A). Consistent with this observation, we found by flow cytometry analysis that Tam-R MCF7 cells were arrested in G1 phase after JQ1 treatment for $24 \mathrm{~h}$, while parental cells were arrested in G1 phase after $>48 \mathrm{~h}$ of JQ1 treatment (Figure $5 \mathrm{C}$ ). Moreover, using these JQ1 target genes, we generated a JQ1-regulated gene signature using the same method as previously described [28]. Using a compendium of several expression array studies, we scored human breast tumors based on the manifestation of the JQ1 gene signature. As shown in Figure 5D, in ER-positive tumors $(N=682)$, high JQ1 signature activity was associated with better patient outcome (log-rank $P=0.001$ ), while in ER-negative tumors $(N=309)$, no survival association was found. These data further support the functional significance of JQ1 in ER signaling in breast cancer.

After 2 days of JQ1 treatment, Tam-R cells began to die, suggesting that prolonged cell cycle arrest may induce apoptosis (Figure 3A). This was confirmed by the appearance of cleaved PARP-1 protein in Tam-R MCF7 cells (Supplementary information, Figure S5B). In con- 


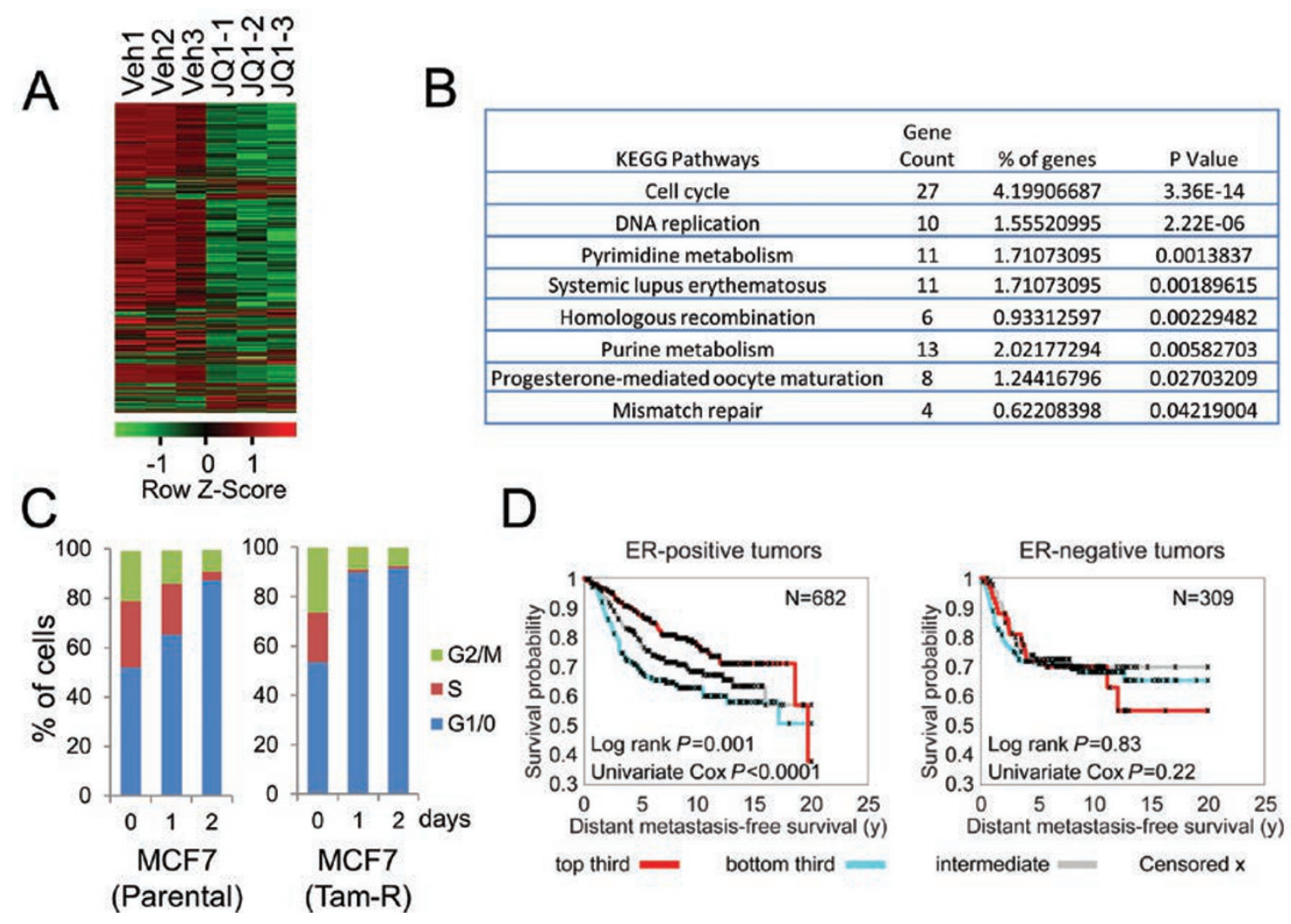

Figure 5 Cellular pathways targeted by JQ1. (A) Heatmap of expression levels for the genes differentially expressed upon treatment with JQ1. Tam-R MCF7 cells were treated with $0.2 \mu \mathrm{M}$ of JQ1 or vehicle (DMSO) for $24 \mathrm{~h}$ before harvest for microarray analysis. (B) Biological pathways were identified by microarray analysis. KEGG pathways were determined by the Database for Annotation, Visualization and Integrated Discovery (DAVID, http://david.abcc.ncifcrf.gov) based on the gene list that is downregulated by JQ1. (C) Tam-R MCF7 cells are more sensitive to JQ1-induced G1 cell cycle arrest. Cells were fixed and stained with propidium iodide (PI) before being analyzed by flow cytometry. (D) Association of the gene expression signature of JQ1 treatment with breast cancer patient survival. For ER-positive and ER-negative subsets, the differences in risk between tumors, according to degree of manifestation of the JQ1 gene signature, is compared using Kaplan-Meier plots (top third, "strong manifestation"; bottom third, "weak manifestation"; middle third, "intermediate manifestation").

trast, parental MCF7 cells did not undergo apoptosis (Supplementary information, Figure S5B).

\section{JQ1 inhibits tumor growth in Tam-R xenograft mouse model when combined with fulvestrant therapy}

Next, we sought to determine the in vivo antitumor activity of JQ1. Ovariectomized nude mice were transplanted subcutaneously with Tam-R MCF7 tumors, and were randomized into two groups when tumor volumes reached $200 \mathrm{~mm}^{3}$. JQ1 or control vehicle was given to these mice by intraperitoneal injection daily. We first performed a pilot experiment to treat the mice for 7 days to test whether JQ1 could downregulate ER $\alpha$ expression in vivo. As shown in Supplementary information, Figure $\mathrm{S} 6 \mathrm{~A}$, the ER $\alpha$ mRNA level was indeed reduced by about $30 \%$ in JQ1-treated tumors; MYC mRNA level was not significantly reduced, although there was a trend. Im- munohistochemical staining confirmed a decreased level of ER $\alpha$ protein in JQ1-treated tumors, and a reduced proliferation rate was observed with Ki67 and histone H3 phospho-Ser10 staining (Supplementary information, Figure S6B). This result demonstrates that JQ1 has in vivo anticancer activity against Tam-R breast cancer. To achieve an optimal drug response in vivo, we tested a combination of JQ1 and fulvestrant/ICI 182,780, an ER $\alpha$ protein degrader, in Tam-R MCF7 xenograft tumors. While single treatment of JQ1 or fulvestrant moderately inhibited tumor growth, the combination of JQ1 and fulvestrant showed a synergistic antitumor activity in the Tam-R tumors. In the vehicle-treated group, the volumes of all the tumors quickly tripled within 17 days, while in the group that received combination therapy, none of the tumors tripled their size after 40 days of treatment, and only about half of the tumors tripled after a prolonged 

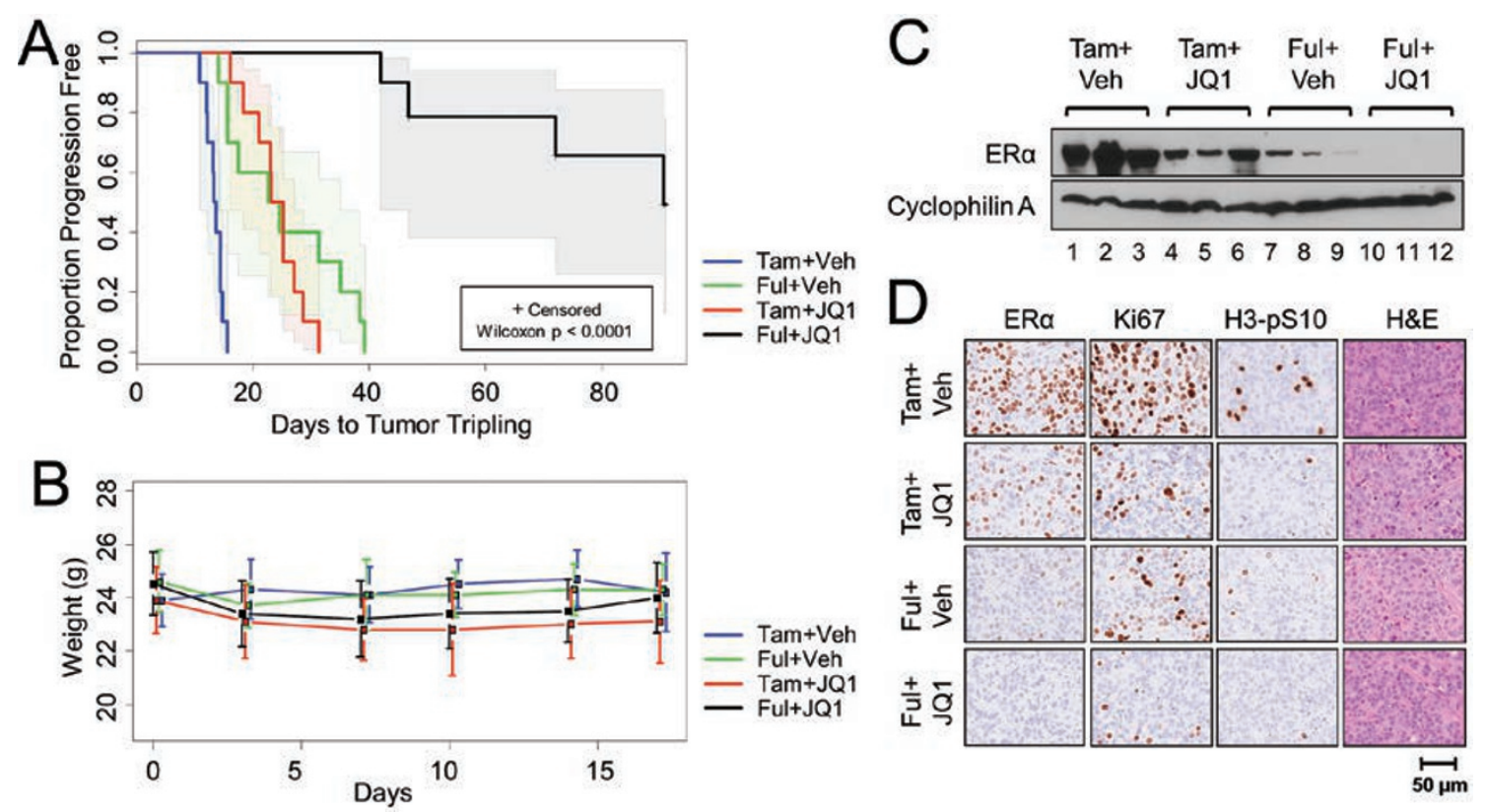

Figure 6 A combination therapy of JQ1 and fulvestrant in Tam-R xenograft mouse model. (A) Ovariectomized mice bearing Tam-R-established MCF7 tumors were randomized (on day 0) into four groups of treatment: Tam+vehicle, Tam+JQ1, fulvestrant+vehicle, and fulvestrant+JQ1, with 10 mice per group. JQ1 was administered daily at $50 \mathrm{mg} / \mathrm{kg}$, while $5 \mathrm{mg}$ fulvestrant was given by subcutaneous injection weekly. Tumors were harvested when they reached $1000 \mathrm{~mm}^{3}$ or 3 months after treatment. (B) Body weight measurement for xenograft experiment shown in A. The error bars show means \pm SEM. (C) Tumors were harvested by the end of the treatment, and western blot was performed using antibodies against ER $\alpha$ and cyclophilin $A$. (D) Immunohistochemical staining of ER $\alpha, \mathrm{Ki} 67$, and histone H3 phospho-Ser10 in xenograft Tam-R tumors from four groups treated with Tam+vehicle, Tam+JQ1, fulvestrant+vehicle, or fulvestrant+JQ1.

treatment (90 days) (Figure 6A). Time-to-tumor tripling from the four groups of treatment was compared using the generalized Wilcoxon test as shown in Supplementary information, Figure S6C. Although JQ1-treated animals lose weight initially and then regain it, generally there is no difference among the four groups in the baseline weights, indicating that JQ1 is well tolerated by mice (Figure 6B). By western blot analysis, the protein levels of ER $\alpha$ were dramatically downregulated in the tumors that received combination therapy of fulvestrant and JQ1 (Figure 6C). Immunohistochemical staining for Ki67 and histone H3 phospho-Ser10 confirmed that the combination therapy potently inhibited the tumor cell proliferation (Figure 6D).

\section{Discussion}

Epigenetics is defined as heritable changes caused by mechanisms other than changes in DNA sequence, including DNA methylation, histone modifications, and noncoding RNA. Epigenomic proteins such as chromatin regulators have emerged as novel therapeutic targets for cancer. For instance, DNA methylase inhibitors, such as
Vidaza and Decitabine, and an HDAC inhibitor, such as Vorinostat, have been used clinically in treating hematological malignancies [29]. Specific inhibitors against H3K79 methylase hDOT1L and H3K27 methylase EZH2 also are being developed for treatment of a variety of cancers [30-32]. The small-molecule inhibitor of epigenomic reader bromodomain JQ1 has shown potent anticancer activity in hematological cancer by targeting the MYC pathway. JQ1 is also thought to be effective in a subset of human lung adenocarcinoma cell lines by inhibiting the expression of an oncogenic transcriptional factor FOSL1 [33]. In a previous report, breast cancer cell lines only show modest sensitivity to JQ1 [34]. In agreement with that observation, we found that the growth of the parental MCF7 and T47D cells was only partially inhibited by JQ1 at high concentrations. Strikingly, the Tam-R cells are more sensitive to JQ1 treatment. In our JQ1 treatment experiment, the ER mRNA level in MCF7 parental cells initially was suppressed significantly but returned to a normal level after prolonged treatment. MYC mRNA levels were only slightly changed by JQ1 in MCF7L parental cells. In contrast, in Tam- $\mathrm{R}$ cells, both ER and MYC mRNA levels were consistently sup- 
pressed by JQ1 even after 3 days of treatment. Our result provides an explanation for the increased JQ1 sensitivity of Tam-R breast cancer cells compared to parental cells, and further indicates that JQ1 targets both ER ad MYC pathways in Tam-R cells. More importantly, JQ1 shows in vivo anticancer activity in suppressing the Tam-R breast cancer growth in the xenograft mouse model. A combination treatment of fulvestrant and JQ1 more effectively downregulated ER $\alpha$ and inhibited in vitro and in vivo tumor growth, providing a new potential approach for treating Tam-R and ER $\alpha$-dependent breast cancer.

\section{Materials and Methods}

\section{Small-scale siRNA screening}

A customized small siRNA library contains individual Stealth RNAi siRNAs (Life Technologies) targeting 29 histone lysine methyltransferases and 18 histone lysine demethylases. All siRNAs used in this study were transfected to MCF7 cells at the final concentration of $20 \mathrm{nM}$ using Lipofectamin RNAiMAX reagent.

\section{Cell culture and transfection}

MCF7, MCF7 RN, T47D, and ZR75-1 cells were maintained in RPMI 1640 medium supplemented with 10\% FCS. MCF7 cells were initially obtained from Dr Marc Lippman in 1985 at the National Cancer Institute, Bethesda, MD. T47D cells were purchased from ATCC. MCF7 RN cells were initially obtained from Dr Robert Nicholson [35]. The Tam-R lines were established from a longtime treatment of $100 \mathrm{nM}$ 4-hydroxy tamoxifen (4-HT) (Sigma) until cell growth was resumed. The parental cells were cultured in RPMI1640 supplemented with 10\% of FCS, whereas their Tam-R derivative line was cultured in phenol red-free medium containing $10 \%$ charcoal-dextran-stripped FCS and $100 \mathrm{nM}$ of 4-HT. All cell lines were authenticated once the resistance was established, and mycoplasma contamination is tested once every 6 months. For estradiol-induced experiments, MCF7 cells were maintained in phenol red-free medium containing 10\% charcoal-dextran-stripped FCS until hormone addition. Fugene 6 transfection reagent (Roche) was used for transient overexpression experiments. All siRNAs used in this study were individual Stealth RNAi siRNAs from Life Technologies, and transfected at the final concentration of 20 nM. Lipofectamin RNAiMAX reagent was used for all the siRNA transfections. The mammalian expression vector of WHSC1/ MMSET (pCEFL-MMSET-II) and its control vector plasmids were kindly provided by Dr Zhenkun Lou at Mayo Clinic [36]. The six different mammalian expression vectors of BRD4 used for deletion mapping in Supplementary information, Figure S2D were obtained from Addgene.

\section{Co-IP and western blot analysis}

Co-IP experiments were done to determine the interaction between WHSC1 and BRD proteins. Two days after transient transfection, 293T cells were harvested and washed with ice-cold phosphate-buffered saline before being disrupted with lysis buffer (50 mM Tris, $100 \mathrm{mM} \mathrm{NaCl}, 0.1 \%$ NP-40, $50 \mathrm{mM} \mathrm{NaF}, 1 \mathrm{mM}$ dithiothreitol, $1 \mathrm{mM}$ phenylmethylsulfonyl fluoride, $1 \mu \mathrm{g} / \mathrm{ml}$ of aprotinin, $0.5 \mu \mathrm{g} / \mathrm{ml}$ of leupeptin, and $0.7 \mu \mathrm{g} / \mathrm{ml}$ of pepstatin). 0.8 mg of protein lysates was incubated with $5 \mu$ l of anti-HA antibody (Roche) at $4{ }^{\circ} \mathrm{C}$ for $4 \mathrm{~h}$, followed by the addition of $10 \mu \mathrm{l}$ of protein G slurry (Santa Cruz Biotechnology) for $1 \mathrm{~h}$. Endogenous IP and co-IP were performed using nuclear extract generated from HeLa cells as previously described [37]. After three washes with lysis buffer, the immunoprecipitated proteins were separated by SDS-PAGE and analyzed by western blot. The antibodies used in co-IP and western blot are anti-BRD2 (Bethyl Labs, A302-583A), anti-BRD3 (Bethyl Labs, A302-368A), anti-BRD4 (Bethyl Labs, A301-985A50), anti-WHSC1 (Abcam, ab75359), anti- $\beta$-actin (Sigma, A2228), anti-ER $\alpha$ (Santa Cruz Biotechnology, sc-543), and anti-cyclophilin A (Cell Signaling Technology, 2175S). The specificity of the BRD antibodies was confirmed by IP/western blot analysis (Supplementary information, Figure S2B). The validation profiles of all the antibodies are available from their company websites.

\section{Chromatin IP}

The ChIP-IT Express kit (Active Motif) was used for ChIP assay in this study following the manufacturer's protocol. The additional antibodies used in ChIP assay were rabbit IgG (Santa Cruz Biotechnology, sc-2027), anti-acetyl-histone H3 (Millipore, 06-599), anti-H3K36me2 (Active Motif, 39255), anti-H3K4me3 (Active Motif, 39159), and anti-H3K36me3 (Active Motif, 61101). These antibodies are all ChIP grade and have been validated by their companies. The ChIP PCR primers for amplification of $E R \alpha / E S R 1$ gene next to the promoter region (A) are: Forward, 5'-CCCACTCAACAGCGTGTCT-3'; Reverse, 5'-CTGCAGGAAAGGCGACAG-3'. The ChIP primers for amplification of $E R \alpha$ gene in the coding region (B) are: Forward, 5'-GAAGAAGCATGGGTAAATGTCA-3'; Reverse, 5'-TCAGCCCTGAACCCAGTG-3'.

\section{$R N A$ isolation and reverse transcription- $q P C R$ analysis}

Total RNA was extracted with TriReagent (Molecular Research Center) (for siRNA screening) or RNeasy Mini Kit (Qiagen). To measure the relative mRNA levels, real-time reverse transcription-PCR (RT-PCR) was performed in an Applied Biosystems 7500 fast real-time PCR system (Applied Biosystems, Foster City, CA). The primers for gene expression assays were designed using online Roche website: https://www.roche-applied-science.com/ sis/rtpcr/upl/index.jsp. The primers for amplication of total $E R \alpha$ : Forward, 5'-ATCCACCTGATGGCCAAG-3'; Reverse, 5'-GCTCCATGCCTTTGTTACTCA-3'. The primers for amplification of specifically ERA7: Forward, 5'-TGCTGGCTACATCATCTCGGTT-3'; Reverse, 5'-CCATGCCTTTGTTACAGAATTAAGCA-3' [24]. The SensiFast SYBR one-step Kit (Bioline) was used for RT-qPCR analysis. For all RT-qPCR experiments in this study, samples were duplicated and the error bars were shown as SEM.

\section{Correlation analysis and microarray analysis}

For the correlation analysis shown in Figure 1E, gene expression and clinical data were downloaded from the TCGA Breast Cancer [38]. Pearson correlation between WHSC1 and ESR1 was computed using the $R$-statistical system within the following subtypes of breast cancer: Luminal A, Luminal B, Her2, and ER positive. For microarray analysis, the RNA was extracted with RNeasy Mini Kit (Qiagen), and the array was performed on GeneChip Human Gene 1.0 ST Array (Affymetrix) at Asuragen Inc. (Austin, 
TX) with triplicated samples. A heatmap was built using the gene expression for the genes differentially expressed upon treatment with JQ1 when a threshold of $\log 2<-0.2$ or $\log 2>0.2$ was applied. Gene expression was transformed by subtracting the mean value and dividing by SD for each individual gene. A heatmap was generated using the $R$-statistical system. Raw microarray data can be accessed at Gene Expression Omnibus (GEO), with accession number GSE49124.

\section{JQ1 signature analysis}

For the "compendium" data set of nine separate breast tumor expression profiling data sets for survival analysis, gene transcription profiling data sets (all on Affymetrix U133 array, A set, and all with DMFS as an outcome measure) were previously obtained from previous studies and consolidated into one data set [39]. Genes within each data set were first normalized to SD from the median, where multiple human array probe sets referenced the same gene, the probe set with the highest variation was used to represent the gene. To score each human breast tumor profile, for similarity to the gene signature of JQ1-treated cells, we derived a " $t$-score" metric for each human tumor in relation to the experimental signature, similar to what we have done in previous analyses [28, 40]; briefly, the $t$-score was defined for each external profile as the two-sided $t$-statistic comparing, within the profile, the average of the genes high in the signature with the average of the genes low in the signature.

\section{MTS assay}

Breast cancer cells were seeded at a density of $2 \times 10^{3}$ cells per well in flat-bottomed 96-well plates (day 0 ) and their growth was measured on days 1, 2, 3, 5, and 7 after JQ1 treatment. Cell media were changed every 2 days. CellTiter 96 AQueous One Solution Reagent (Promega) was added to each well following the manufacturer's instructions. After $1 \mathrm{~h}$ of incubation, the cell viability was determined by measuring the absorbance at $490 \mathrm{~nm}$ using the Multiskan FC microplate photometer (Thermo Scientific). For all the MTS assays done in this study, samples were treated in quadruplicate and error bars were shown as SEM.

\section{Apoptosis assay}

Parental and Tam-R MCF7 cells were treated with various dosages $(0.2,0.5,1 \mu \mathrm{M})$ of JQ1 for 2 days followed by being harvested for western blot. The cleaved ( $89 \mathrm{kDa}, \mathrm{C}$ terminus) and full-length (116 kDa) forms of PARP-1 protein were detected by PARP-1 antibody (Santa Cruz Biotechnology, sc-8007, dilution $1: 200)$.

\section{In vivo Tam-R breast cancer xenograft studies and immuno-} histochemistry

The antitumor effect of JQ1 and JQ1/fulvestrant was evaluated in Tam-R breast cancer xenograft mouse model. Briefly, tamoxifen citrate-treated 4-5-week-old ovariectomized athymic mice were subcutaneously implanted with Tam-R breast tumor fragments at the hypogastrium area. Three-five weeks later when the tumor size reaches $150-200 \mathrm{~mm}^{3}$, mice were randomized into four treatment groups by simple randomization method, totally 10 mice per group. The allocation started from the first to the fourth group, and then from the fourth to the first group for the next round. Unless any animals die from an unknown reason, no animal has been ex- cluded from the study. JQ1 or DMSO (vehicle) was administered daily at $50 \mathrm{mg} / \mathrm{kg}$ with $10 \%$ hydroxypropyl beta-cyclodextrin solution as a carrier, while $5 \mathrm{mg}$ fulvestrant was given by subcutaneous injection weekly [41]. Tumors were monitored and tumor volumes and body weight were measured twice a week. Blind measurements were carried out to avoid unconscious biases. Tumors were harvested for molecular studies after 3 months of treatment or when they reached the size of $1000 \mathrm{~mm}^{3}$. Small pieces of the tumors were fixed and embedded in paraffin, and additional materials were kept at $-80{ }^{\circ} \mathrm{C}$. H\&E staining was performed to examine the overall structure of the tumors, and the cell proliferation was determined by immunohistochemical staining of Ki67 and phosphorylated histone $\mathrm{H} 3$ at Ser10. The antibodies used were histone H3 phospho-Ser10 (Millipore 06-570, 1:300), Ki67 (Dako M7240, 1:200), and ER $\alpha$ (Vector Laboratories VP-E613, 1:200). Other tumor parts were used for RNA extraction and protein preparation. All in vivo animal studies were conducted under a protocol approved by Institutional Animal Care and Use Committees (IACUC) at the Baylor College of Medicine.

\section{Acknowledgments}

We thank our colleagues Drs Ming-Jer Tsai, Sophia Tsai, and David Lonard for helpful discussion, and Tamika Mitchell and Dr Vincent Yu for technical support. We also thank Dr Zhenkun Lou (Mayo Clinic) for providing the WHSC1 expression vector. This work was supported by R01HD08188 (BWO), DoD BCRP BC122115 (QF), K01DK084209 (QF), Komen Promise Grant (BWO and CKO), R01HD07857 (BWO), CPRIT RP100348, DOD-BC120894 (BWO), Clayton Foundation, ACS RSG1306101TBE (BH), Dunn Foundation, Breast Cancer Research Foundation (CKO and RC), and P30CA125123 (SGH).

\section{References}

1 Clarke R, Liu MC, Bouker KB, et al. Antiestrogen resistance in breast cancer and the role of estrogen receptor signaling. Oncogene 2003; 22:7316-7339.

2 Ross-Innes CS, Stark R, Teschendorff AE, et al. Differential oestrogen receptor binding is associated with clinical outcome in breast cancer. Nature 2012; 481:389-393.

3 Lupien M, Meyer CA, Bailey ST, et al. Growth factor stimulation induces a distinct ER(alpha) cistrome underlying breast cancer endocrine resistance. Genes Dev 2010; 24:2219-2227.

4 Mayer I. Role of mTOR inhibition in preventing resistance and restoring sensitivity to hormone-targeted and HER2-targeted therapies in breast cancer. Clin Adv Hematol Oncol 2013; 11:217-224.

5 Munster PN, Thurn KT, Thomas S, et al. A phase II study of the histone deacetylase inhibitor vorinostat combined with tamoxifen for the treatment of patients with hormone therapy-resistant breast cancer. Br J Cancer 2011; 104:1828-1835.

6 Osborne CK, Bardou V, Hopp TA, et al. Role of the estrogen receptor coactivator AIB1 (SRC-3) and HER-2/neu in tamoxifen resistance in breast cancer. J Natl Cancer Inst 2003; 95:353-361.

7 Osborne CK, Schiff R. Mechanisms of endocrine resistance in breast cancer. Annu Rev Med 2011; 62:233-247. 
8 Huang Y, Nayak S, Jankowitz R, Davidson NE, Oesterreich S. Epigenetics in breast cancer: what's new? Breast Cancer Res 2011; 13:225.

9 Kuo AJ, Cheung P, Chen K, et al. NSD2 links dimethylation of histone $\mathrm{H} 3$ at lysine 36 to oncogenic programming. Mol Cell 2011; 44:609-620.

10 Nimura K, Ura K, Shiratori H, et al. A histone H3 lysine 36 trimethyltransferase links Nkx2-5 to Wolf-Hirschhorn syndrome. Nature 2009; 460:287-291.

11 Hudlebusch HR, Santoni-Rugiu E, Simon R, et al. The histone methyltransferase and putative oncoprotein MMSET is overexpressed in a large variety of human tumors. Clin Cancer Res 2011; 17:2919-2933.

12 Kassambara A, Klein B, Moreaux J. MMSET is overexpressed in cancers: link with tumor aggressiveness. Biochem Biophys Res Commun 2009; 379:840-845.

13 Ivshina AV, George J, Senko O, et al. Genetic reclassification of histologic grade delineates new clinical subtypes of breast cancer. Cancer Res 2006; 66:10292-10301.

14 Ginestier C, Cervera N, Finetti P, et al. Prognosis and gene expression profiling of 20q13-amplified breast cancers. Clin Cancer Res 2006; 12:4533-4544.

15 Malovannaya A, Lanz RB, Jung SY, et al. Analysis of the human endogenous coregulator complexome. Cell 2011; 145:787-799.

16 O’Malley BW, Malovannaya A, Qin J. Minireview: nuclear receptor and coregulator proteomics--2012 and beyond. Mol Endocrinol 2012; 26:1646-1650.

17 Sarai N, Nimura K, Tamura T, et al. WHSC1 links transcription elongation to HIRA-mediated histone H3.3 deposition. EMBO J 2013; 32:2392-2406.

18 Wu SY, Chiang CM. The double bromodomain-containing chromatin adaptor Brd4 and transcriptional regulation. J Biol Chem 2007; 282:13141-13145.

19 Dawson MA, Prinjha RK, Dittmann A, et al. Inhibition of BET recruitment to chromatin as an effective treatment for MLL-fusion leukaemia. Nature 2011; 478:529-533.

20 Mochizuki K, Nishiyama A, Jang MK, et al. The bromodomain protein Brd4 stimulates $\mathrm{G} 1$ gene transcription and promotes progression to S phase. J Biol Chem 2008; 283:9040-9048.

21 Delmore JE, Issa GC, Lemieux ME, et al. BET bromodomain inhibition as a therapeutic strategy to target c-Myc. Cell 2011; 146:904-917.

22 Herrmann H, Blatt K, Shi J, et al. Small-molecule inhibition of BRD4 as a new potent approach to eliminate leukemic stem- and progenitor cells in acute myeloid leukemia AML. Oncotarget 2012; 3:1588-1599.

23 Zuber J, Shi J, Wang E, et al. RNAi screen identifies Brd4 as a therapeutic target in acute myeloid leukaemia. Nature 2011; 478:524-528.

24 Groenendijk FH, Zwart W, Floore A, Akbari S, Bernards R. Estrogen receptor splice variants as a potential source of false-positive estrogen receptor status in breast cancer diagnostics. Breast Cancer Res Treat 2013; 140:475-484.

25 Loven J, Hoke HA, Lin CY, et al. Selective inhibition of tumor oncogenes by disruption of super-enhancers. Cell 2013; 153:320-334.

26 Ott CJ, Kopp N, Bird L, et al. BET bromodomain inhibition targets both c-Myc and IL 7R in high-risk acute lymphoblastic leukemia. Blood 2012; 120:2843-2852.

27 Eeckhoute J, Keeton EK, Lupien M, Krum SA, Carroll JS, Brown M. Positive cross-regulatory loop ties GATA-3 to estrogen receptor alpha expression in breast cancer. Cancer Res 2007; 67:6477-6483.

28 Cancer Genome Atlas Research Network. Integrated genomic analyses of ovarian carcinoma. Nature 2011; 474:609-615.

29 Suarez L, Gore SD. Demethylation demystification. Blood 2013; 121:1488-1489.

30 Daigle SR, Olhava EJ, Therkelsen CA, et al. Selective killing of mixed lineage leukemia cells by a potent small-molecule DOT1L inhibitor. Cancer Cell 2011; 20:53-65.

31 McCabe MT, Ott HM, Ganji G, et al. EZH2 inhibition as a therapeutic strategy for lymphoma with EZH2-activating mutations. Nature 2012; 492:108-112.

32 Knutson SK, Wigle TJ, Warholic NM, et al. A selective inhibitor of EZH2 blocks H3K27 methylation and kills mutant lymphoma cells. Nat Chem Biol 2012; 8:890-896.

33 Lockwood WW, Zejnullahu K, Bradner JE, Varmus H. Sensitivity of human lung adenocarcinoma cell lines to targeted inhibition of BET epigenetic signaling proteins. Proc Natl Acad Sci USA 2012; 109:19408-19413.

34 Mertz JA, Conery AR, Bryant BM, et al. Targeting MYC dependence in cancer by inhibiting BET bromodomains. Proc Natl Acad Sci USA 2011; 108:16669-16674.

35 Knowlden JM, Hutcheson IR, Jones HE, et al. Elevated levels of epidermal growth factor receptor/c-erbB2 heterodimers mediate an autocrine growth regulatory pathway in tamoxifen-resistant MCF7 cells. Endocrinology 2003; 144:10321044.

36 Pei $\mathrm{H}$, Zhang L, Luo K, et al. MMSET regulates histone H4K20 methylation and 53BP1 accumulation at DNA damage sites. Nature 2011; 470:124-128.

37 Feng Q, Yi P, Wong J, O’Malley BW. Signaling within a coactivator complex: methylation of SRC-3/AIB1 is a molecular switch for complex disassembly. Mol Cell Biol 2006; 26:7846-7857.

38 Cancer Genome Atlas Network. Comprehensive molecular portraits of human breast tumours. Nature 2012; 490:61-70.

39 Kessler JD, Kahle KT, Sun T, et al. A SUMOylation-dependent transcriptional subprogram is required for Myc-driven tumorigenesis. Science 2012; 335:348-353.

40 Gibbons DL, Lin W, Creighton CJ, et al. Expression signatures of metastatic capacity in a genetic mouse model of lung adenocarcinoma. PLoS One 2009; 4:e5401.

41 Massarweh S, Osborne CK, Jiang S, et al. Mechanisms of tumor regression and resistance to estrogen deprivation and fulvestrant in a model of estrogen receptor-positive, HER-2/ neu-positive breast cancer. Cancer Res 2006; 66:8266-8273.

(Supplementary information is linked to the online version of the paper on the Cell Research website.)

(c) (i) () - This work is licensed under the Creative Commons Attribution-NonCommercial-No Derivative Works 3.0 Unported License. To view a copy of this license, visit http:// creativecommons.org/licenses/by-nc-nd/3.0 